\title{
The Non-Dominant Hand in a Swedish Sign Language Discourse
}

\author{
Anna-Lena Nilsson
}


Acknowledgement: Based on "The non-dominant hand in a Swedish Sign Language discourse." In Vermeerbergen, M., Leeson, L. \& Crasborn, O.: Simultaneity in Signed Languages: Form and Function. Currrent Issues in Linguistic Theory Volume 281. 2007. pp 163-185. With kind permission by John Benjamins Publishing Company, Amsterdam/Philadelphia.

www.benjamins.com 


Contents 12918





\section{Introduction ${ }^{1}$}

Signers have two hands at their disposal to produce signs, and a stretch of signed discourse usually contains both one-handed and two-handed signing. As was pointed out by Battison regarding American Sign Language (ASL) "most people with strong hand preference will use their dominant hand to play the active role, and their non-dominant hand in the static role" (1974:8). The dominant hand will thus be used to produce most one-handed signs and fingerspellings, be the more active hand in asymmetric two-handed signs, and function as one of the active hands in symmetrical two-handed signs. The non-dominant hand will not be inactive though. Among its easily discernible functions are (1) being the base hand (Klima \& Bellugi 1979:64) in asymmetric two-handed signs, (2) being one of the articulators in symmetric two-handed signs, and (3) to produce signs of its own. There are also instances where the non-dominant hand anticipates or perseverates from twohanded signs.

In the following, an attempt is made to describe the activities of the nondominant hand when it does not participate in the production of a twohanded sign. The aim is thus not to describe e.g. the internal structure of two-handed signs or phonological processes within individual signs; several such studies have been published regarding different signed languages, e.g. Battison (1974) for ASL. Modified forms of signs, where the non-dominant hand is active due to some morphological process expressing, for example, plurality and/or repeated action (see e.g. Klima \& Bellugi (1979) for ASL; Bergman (1983) for Swedish Sign Language) will not be included either. Constructions known as polysynthetic signs in descriptions of Swedish Sign Language (e.g. Wallin 2000), or as classifier constructions (e.g. Emmorey 2003), are also excluded.

The fact that signed languages permit simultaneous production of distinct elements by the two hands has frequently been observed. In a paper including studies covering five different signed languages (published from1975 to the mid-nineties), Miller focuses on "non-classifier constructions involving the simultaneous production of distinct signs" (1994:89). Simultaneous constructions such as, e.g. an enumeration morpheme, object incorporation,

${ }^{1}$ I would like to thank Lena Johansmide for willingly allowing me to use this recording of her, and my colleagues at Stockholm University for interesting discussions about the material. I owe special gratitude to Brita Bergman for invaluable help in preparing this text. 
(topic) perseveration and Index $x_{\text {pro }}$ are discussed, and they are also compared to similar constructions in Quebec Sign Language (LSQ). Some of the activities of the non-dominant hand described in the present study are discussed by Miller too, but from a different perspective and using different terminology.

Stretching the point, one could hypothesize that whenever the dominant hand produces a one-handed sign, the non-dominant hand is not required to take part in the production of that sign and thus will be inactive. No detailed analysis is needed, however, to note that the non-dominant hand is not inactive to such a large extent in normal signed discourse. In this study, the activities of the non-dominant hand in a whole discourse are described, something which, to the best of my knowledge is not found in any other study. As we will see, simultaneity is a key concept and there are several factors contributing to a markedly two-handed impression of this signed discourse. 


\section{Material and method}

The material analyzed is a Swedish Sign Language discourse, consisting of a video recorded monologue ( 9 minutes 44 seconds long) where a deaf woman retells the contents of an autobiography she has read - Livets hjul: En självbiografi $i$ dödens närhet. (original title Wheel of life.) - written by Elisabeth Kübler-Ross. There is a listener (a native signer) sitting by the camera.

In order to describe the activities of the non-dominant hand when it is not part of a two-handed sign, a list was made of the signs that occur in the material which are considered one-handed in citation form. The total number of occurrences of one-handed signs is 613 , counting all instances regardless of whether the same sign has already previously occurred or not. The total number of instances of two-handed signs is 538, making the total number of instances of signs 1151 . Thus, approximately $53 \%$ of the instances of signs are one-handed, and approximately $47 \%$ two-handed in citation form. This can be compared to the Swedish Sign Language dictionary (Svenskt teckenspråkslexikon, 1997) where approximately $45 \%$ of the main entries are onehanded signs.

Having identified all instances of one-handed signs, the activities of the non-dominant hand during the production of these signs were analyzed, and categorized. In the following, the activities of the non-dominant hand will be presented, starting with those where it is least active and adds least to the content of the discourse, and gradually moving towards more active participation and contribution to content. (All percentages have been rounded off to the nearest whole number.) 


\section{The non-dominant hand}

\section{In lap}

There are sequences in the discourse where the non-dominant hand does not participate in the production of signs, but rests in the signer's lap. This position, illustrated in Figure 1, is referred to as 'in lap'. ${ }^{2}$ Short sequences with 'in lap' occur at irregular intervals in the discourse, lasting one or a few signs. There are also seven longer sequences, one is $26 \mathrm{sec}$., where the nondominant hand rests in the signer's lap during the one-handed signs, but actively takes part in the production of two-handed signs when they occur. Of the one-handed signs in the discourse, $19 \%(n=116)$ co-occur with 'in lap'.

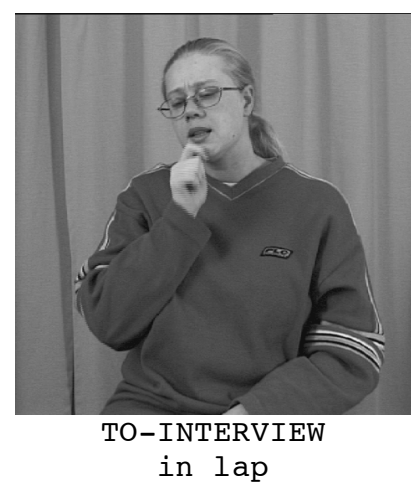

Figure 1: Illustration of 'in lap'.

\footnotetext{
${ }^{2}$ Transcriptions consist of two lines, with or without photos to illustrate the signs. The top line represents the right (dominant) hand, the bottom line the left (non-dominant) hand. Glosses in capitals are used to identify Swedish Sign Language signs. Some glosses indicate the form of the sign, or the function, whereas most are chosen to represent the meaning of the sign. That does not imply that the sign has the same semantic, morphological or syntactic characteristics as the English word chosen. Glosses with hyphens between them are used when more than one word is used to identify a sign, glosses with a slash (/) between them are used to indicate compound signs. A gloss preceded by a ' $\#$ ' indicates a fingerspelled item. A line of hyphens (----) is used to indicate the duration of a sign, when that is of particular interest. Lower-case letters after glosses are used to indicate the direction of the movement of a sign; f-forward, fl-forward left, fr-forward right. The English translations below transcriptions give the meaning in that specific context.
} 


\section{At chest}

Another rest position of the non-dominant hand entails it being held still at chest height in a loose fist configuration. Sometimes the non-dominant hand is quite relaxed in this position, with a tendency for the index finger of this lax hand to be slightly extended. Two examples of 'at chest' are illustrated in Figure 2. Different forms of this position co-occur with a total of $22 \%(n=$ 133) of the one-handed signs in the material.
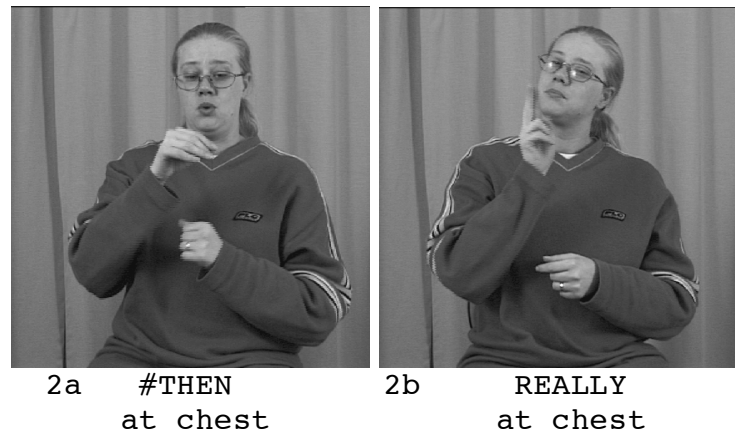

Figure 2: Illustrations of 'at chest'.

\section{Mirror at chest}

In the positions of the non-dominant hand described so far, it is held still and the handshape is not influenced by that of the dominant hand. In 'mirror at chest', the non-dominant hand is also held still, in a position similar to that of 'at chest', but it mirrors the handshape and orientation of the dominant hand. ${ }^{3}$ In the example in Figure 3, we see the one-handed sign GRADUATE, where the dominant hand is held at the contralateral side of the signer's chest, assuming a hand configuration where the thumb, index finger and middle finger are extended. The non-dominant hand is held still at chest height, but assumes the same handshape and orientation as the dominant hand. Since the non-dominant hand does not move the co-articulation is only partial. 'Mirror at chest' co-occurs with $6 \%(n=37)$ of the one-handed signs.

'In lap', 'at chest', and 'mirror at chest' can all be described as rest positions of the non-dominant hand, since it does not participate in the sign production, and does not add to the content of the discourse. For 'at chest' and 'mirror at chest', however, the fact that the non-dominant hand does not rest

\footnotetext{
${ }^{3}$ Sandler (1993) also uses the term mirror in her paper on sign language phonology (ASL), but in a different way. According to her the non-dominant hand has two roles. "In one role, it is a mirror of the dominant hand, what I call an echo articulator. In the other role, it is not an articulator at all, but a place of articulation, like the head or the trunk" (1993:337-338).
} 
in the signer's lap but is held at chest height makes it appear to take part in the discourse production, something we will return to in the discussion below.

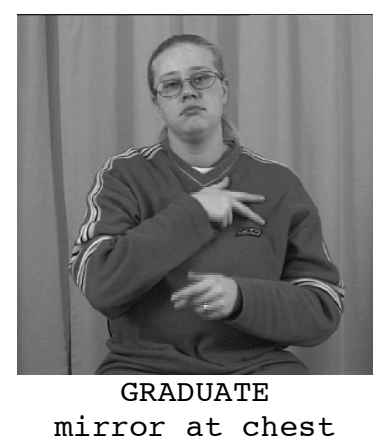

Figure 3: Illustration of 'mirror at chest'.

\section{Mirroring}

In the rest positions of the non-dominant hand discussed in the previous sections, the non-dominant hand is held still, even though its position may contribute to a two-handed impression of the signing. In Figure 4, as the dominant hand produces the one-handed sign TO-FAIL, the non-dominant hand not only assumes the same handshape and orientation, but also performs the same movement (both the hand internal movement and the downward path movement) at a slightly lower location. This full co-articulation makes this instance of TO-FAIL look like a two-handed sign produced with the non-dominant hand slightly lower than the dominant. Even though the non-dominant hand is active here, this is a purely phonetic, sign-internal process, which does not add any content to the discourse. 'Mirroring' is the term used for instances of signs in the material which are considered onehanded in citation form, but are produced in this way. Of the one-handed signs in the discourse, $4 \%(n=22)$ co-occur with 'mirroring'. 


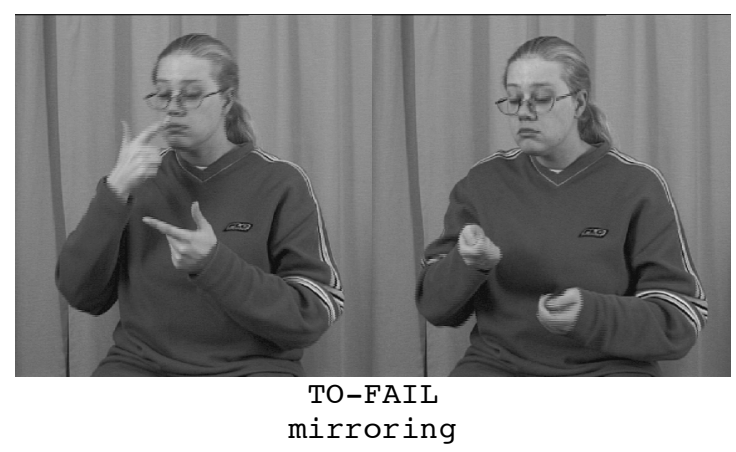

Figure 4: Illustration of 'mirroring'.

\section{Doubling}

The material also contains instances of signs that are one-handed in citation form, but produced with both hands active and performing the same movement at the same height. This is referred to as 'doubling', and there are two such instances in Figure 5 below (which also illustrates other activities of the non-dominant hand), viz. the first and third signs in the first row. Both HOW and TO are one-handed in citation form, but in this example they are produced with 'doubling'. Instances of 'doubling', where we see active participation of the non-dominant hand, all occur in stretches of signing that are produced with a certain kind of intensity. In these stretches of signing, all signs, including signs that are one-handed in citation form, are produced with two hands. The intensity in the sign production combined with the twohanded sign production seems to reinforce what is said. There are 15 instances of 'doubling', co-occurring with $2 \%$ of the one-handed signs.

\section{Sign fragments}

'Sign fragments' are instances of a special kind of perseveration, where the non-dominant hand has been part of the production of a two-handed sign, and then remains in that position while the dominant hand continues to produce signs. Whereas phonological perseveration is non-meaningful, sign fragments are meaningful and indicate to the listener who/what the topic of the continued discourse is. In Figure 5 (below), the signer has told us that it is when people know they are soon going to die that they truly feel alive. This discourse topic is then kept constant with a fragment of the last sign LIVE (first sign in the last row of photos) on the non-dominant hand. Then 
the dominant hand produces the sign VIEW-OF-SOMETHING, which starts from the left and moves towards (and actually past) the sign fragment of LIVE.

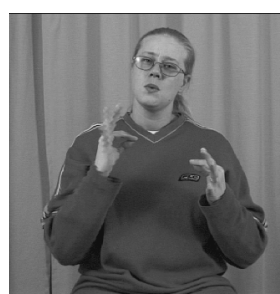

HOW doubling

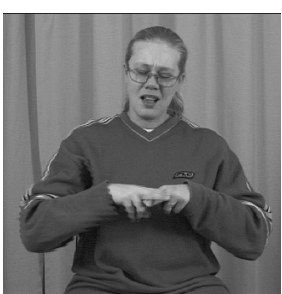

FEEL

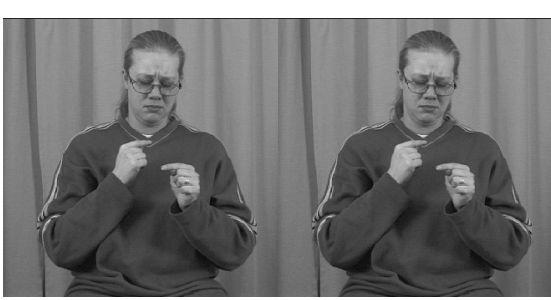

GET-NEARER

POINT-G---

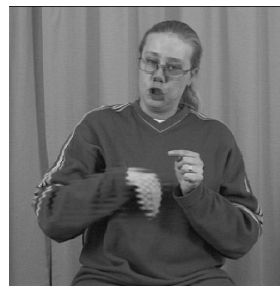

DIE

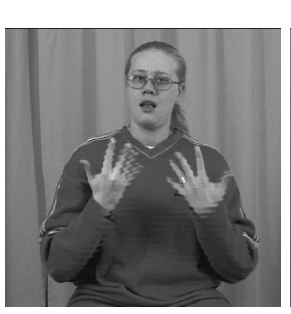

LIVE

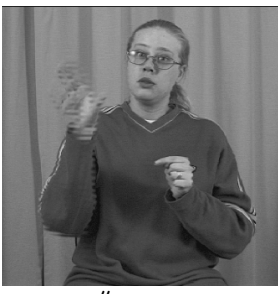

\#THEN

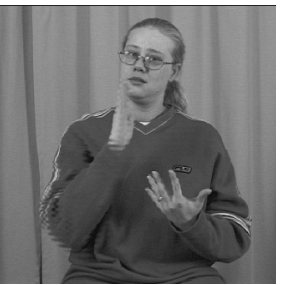

POSS

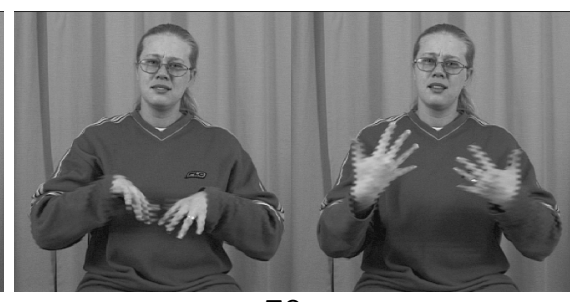

TO

doubling

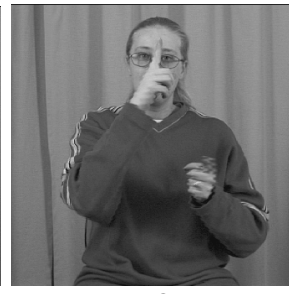

PEOPLE

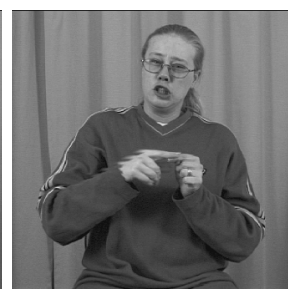

SOON

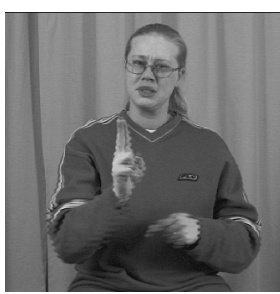

REALLY

at chest

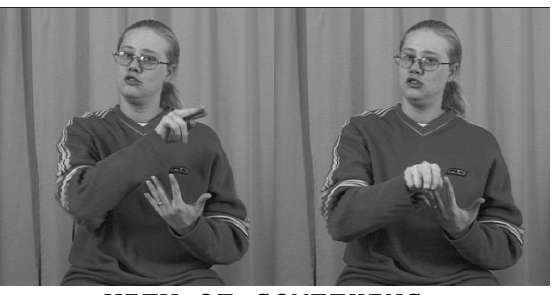

VIEW-OF-SOMETHING

What it feels like to live and know you are soon going to die - that's when people are truly alive. That is her view of it.

Figure 5: Illustration of 'doubling', 'POINT-G, 'at chest', and a 'sign fragment'. 
The direction where VIEW-OF-SOMETHING starts has previously been used for reference to the main character of the story, and we thus know that it is her view that is told.

The analyzed material contains ten sequences with 'sign fragments', which all remain from symmetrical two-handed signs, co-occurring with $6 \%$ $(n=37)$ one-handed signs. No instances of 'sign fragments' from asymmetric two-handed signs have been found.

Similar use of the non-dominant hand has been noted in many studies of signed languages, using varying terminology and accounting for it in different ways. The use of the non-dominant articulator "to perform syntactic/semantic functions during simultaneous articulation" is one of the areas covered in Friedman (1975:953). Among the functions mentioned are e.g. maintaining topic or focus, maintaining locative, temporal or pronominal reference, and emphatic or contrastive stress. Gee \& Kegl (1983:246), also for ASL, describe how a sign can perseverate on the non-dominant hand "as topic or background information for a number of lines", claiming that this is "an interesting device for topic marking and topic chaining unique to ASL". Ahlgren \& Bergman (1994) have observed a phenomenon in Swedish Sign Language that they termed 'referential cue', which also involves maintaining a weak hand position to indicate the identity of a referent. (Also cf. Miller 1994, (topic) perseveration,mentioned above.)

Liddell (2003) uses the term 'sign fragments' for instances when the weak hand maintains its configuration from a preceding sign, while the strong hand continues to produce a one-handed sign, and this remaining 'sign fragment' does not appear to serve any semantic function. According to Liddell the signer can choose to assign semantic significance to a fragment of a preceding sign, though, in which case the fragment is no longer meaningless but constitutes a fragment buoy. (Buoys are discussed in the next section). In ASL, this is done by directing the index finger and the eye gaze at the fragment. The 'sign fragments' in the material of this study add to the content of the discourse, but as opposed to Liddell's fragment buoys they are not pointed at, and the gaze direction varies as a result of whether the story is told from the signer's perspective or from the perspective of a discourse participant. What also distinguishes these meaningful 'sign fragments' from other signs in this Swedish Sign Language discourse that indeed are treated as buoys, is that they are the result of the nondominant hand remaining in position from several different two-handed signs, and thus do not have a fixed form.

\section{Buoys}

Whereas the 'sign fragments' described in the previous section were instances of a special type of perseveration, this section will look at signs that are independently produced by the non-dominant hand. The concept of a 
'buoy' is introduced by Liddell (2003), in his description of American Sign Language. Buoys are signs produced with the weak hand, and held in a stationary configuration as the strong hand continues to produce signs. Semantically, buoys "help guide the discourse by serving as conceptual landmarks as the discourse continues" (2003:223). In the following sections, several types of signs in the material, produced by the non dominant hand while the dominant hand continues to sign, will be compared to some of the buoys discussed by Liddell. ${ }^{4}$

\section{The POINTER buoy}

In Figure 6, a pointing sign on the non-dominant hand is directed to the left (earlier marked for reference to the main character of the story), and is then held stationary while the dominant hand produces the one-handed sign WOW. The next sign is the two-handed sign STRANGE. In citation form, the handshape of the base hand would be either a fist or the flat hand. Here the nondominant hand maintains the index finger handshape of the pointing sign, but the orientation of the hand is changed to that which the stationary hand would have in the citation form of STRANGE. The non-dominant hand then resumes its direction to the left, and it is kept like that while the one-handed sign CHILDHOOD is produced, and also during the sign TOO, which in citation form is a two-handed symmetrical sign.

The form and use of the pointing sign in Figure 6 is similar enough to that of a sign in ASL that Liddell (2003) describes and refers to as the POINTER buoy, to warrant the use of the term POINTER buoy in Swedish Sign Language too. ${ }^{5}$ It is the only buoy that actually points, and the non-dominant hand points toward an important discourse element, thereby directing attention to it, while the dominant hand continues to sign. This buoy co-occurs with $3 \%$ of the one-handed signs $(n=20)$.

The POINTER buoy in ASL frequently has a palm-down orientation, though, whereas in Swedish Sign Language the ulnar side of the hand is directed down when it is produced in the horizontal plane. ${ }^{6}$ In this Swedish Sign Language discourse, the dominant hand can either produce one-handed signs (also one-handed variants of normally two-handed symmetric signs) while the POINTER is held, or the dominant hand can be active in a twohanded asymmetric sign, acting on the radial side of the non-dominant hand

\footnotetext{
${ }^{4}$ For a more thorough discussion of buoys see Liddell, Vogt-Svendsen \& Bergman in this volume.

${ }^{5}$ This pointing sign in Swedish Sign Language was first described as TEMA-PEK (THEMEINDEX), in lecture notes by Brita Bergman in 2001.

${ }^{6}$ The only instance with more of a palm-down orientation in the material of this study is one where the THEME buoy is not produced in the horizontal plane, but directed forward and upward, with the palm directed slightly forward/downward.
} 
when it produces this pointing sign. ${ }^{7}$ In Figure 6 the orientation of the nondominant hand as base hand actually changes so much that it can hardly be claimed to still produce the POINTER buoy.

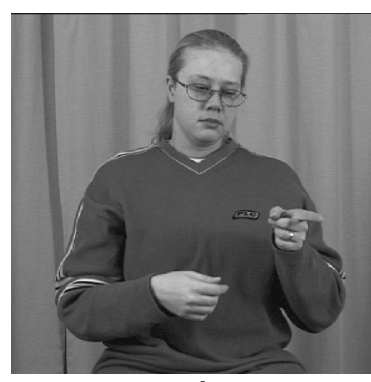

at chest

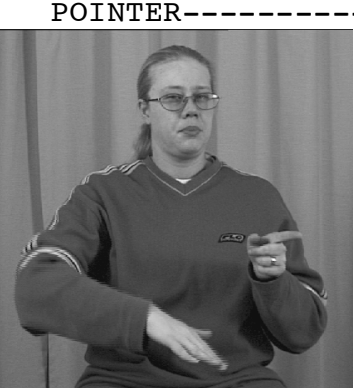

CHILDHOOD

POINTER-1

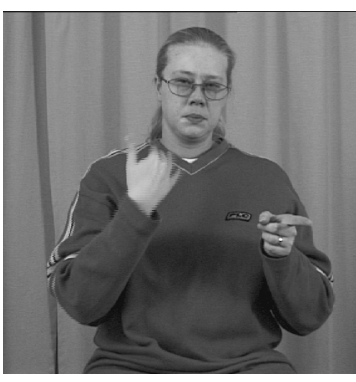

WOW

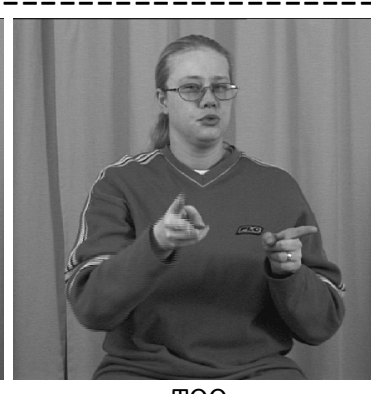

TOO

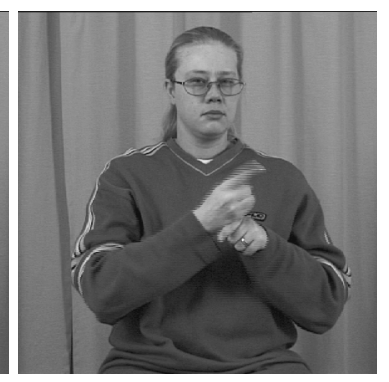

STRANGE

She had a really strange childhood too.

Figure 6: Use of the 'POINTER buoy'.

\section{The THEME buoy}

In Figure 7 below, the signer is introducing an important discourse theme patients dying from cancer. The first two signs, NON-1ST-SING. and \#SHE are directed forward, a direction that is now introduced for reference to the main character of the story. A hand configuration with a raised index finger then appears on the non-dominant hand, and is held stationary while the signer produces the perfect marker PERF. ${ }^{8}$ and the one-handed sign TO-INTERVIEW with the dominant hand. The next two signs MANY and PATIENT are twohanded, and both hands are used to produce them. During the following onehanded sign CL-PERSON the handshape on the non-dominant hand changes to something resembling a pointing sign, but analyzed as an instance of the rest

\footnotetext{
${ }_{8}^{7}$ Also noted in Liddell, Vogt-Svendsen \& Bergman (2004).

${ }^{8}$ The perfect marker is two-handed in citation form, but often produced with only the dominant hand.
} 
position 'at chest'. The next sign, ESPECIALLY, is two-handed and produced with both hands. It is followed by two one-handed signs: CANCER and NON1ST-PLUR, and while they are produced by the dominant hand the raised index finger re-occurs on the non-dominant hand. It disappears again, however, during the next three, two-handed, signs, which are not shown in the example.

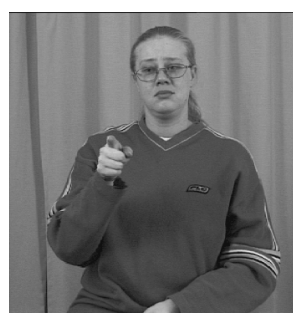

NON-1ST-SING .

\#SHE

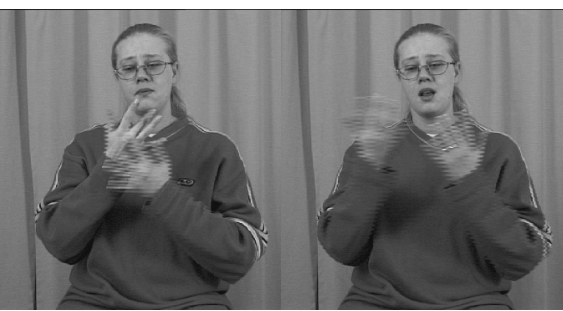

MANY

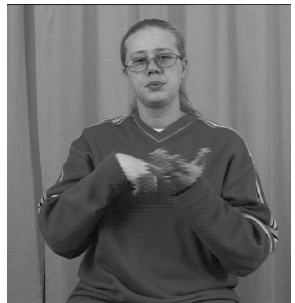

ESPECIALLY

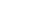

CANCER
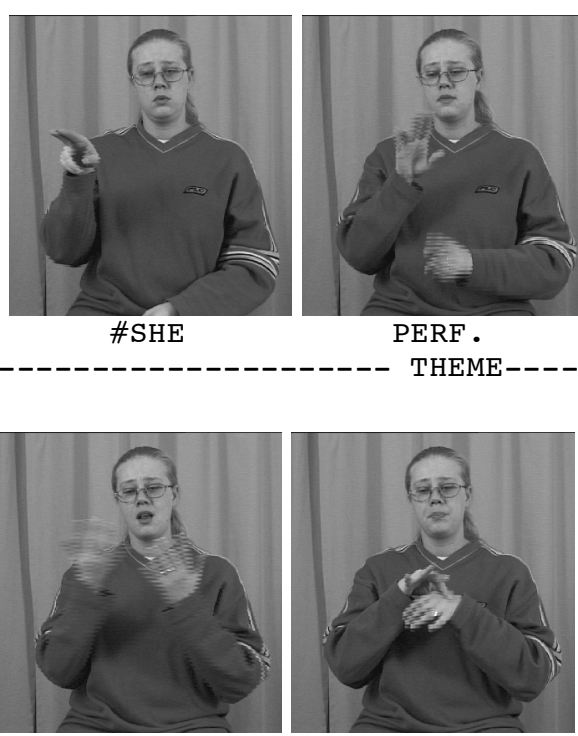

PATIENT

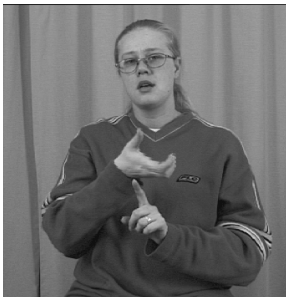

THEME-
PERF .

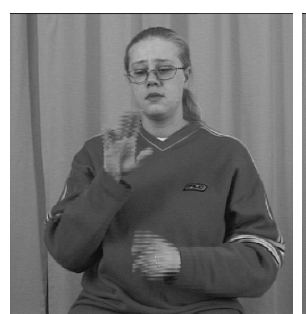

TO-INTERVIEW
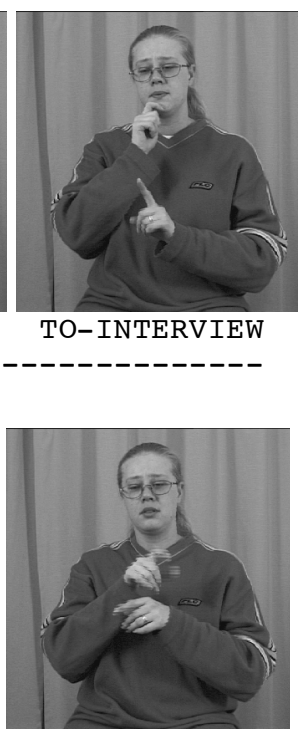

CL-PERSON

at chest

She has interviewed many patients, especially cancer patients...

Figure 7: Use of the 'THEME buoy'.

As can be seen in Figure 8, this discourse theme is mentioned again approximately five minutes later in the discourse, and the raised index finger re-occurs on the non-dominant hand precisely while the sign CANCER is produced with the dominant hand. 


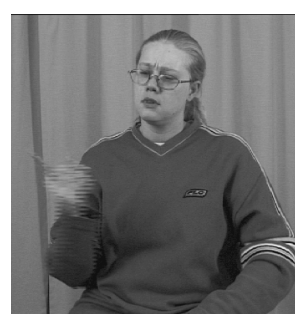

NON-1ST-SING . in lap

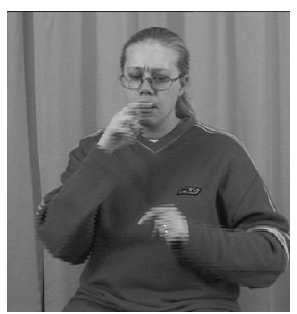

DO-RESEARCH

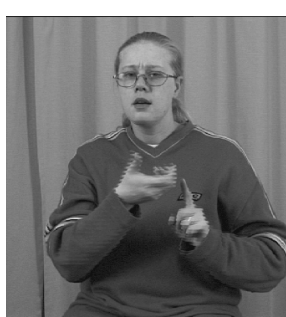

CANCER

THEME

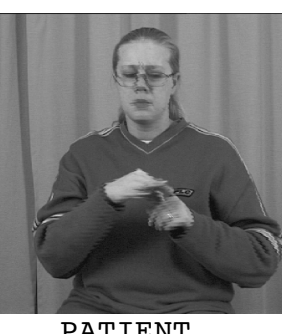

PATIENT

She had done research on cancer patients...

Figure 8: The 'THEME buoy' reappears five minutes later in the discourse.

Both the form and the use of this raised index finger on the non-dominant hand very much resemble the THEME buoy in ASL, which is a buoy whose presence "signifies that an important discourse theme is being discussed" (Liddell 2003:242). The form and meaning of this buoy blend to produce a visible representation of that discourse theme. Liddell (2003:246) also has an example where the THEME buoy reappears after approximately five minutes. The Swedish Sign Language THEME buoy co-occurs with 5\% $(n=32)$ of the one-handed signs.

The ASL THEME buoy can serve as the base hand in an asymmetrical twohanded sign (Liddell 2003:245). However, in this Swedish Sign Language discourse, the THEME buoy disappears when the signer produces two-handed signs. It is thus not used as base hand, but replaced by the two-handed sign. As the ASL sign, the Swedish Sign Language sign may have pronominal signs directed at it, but the signer can also actually touch it, something that is not mentioned by Liddell.

\section{List buoys}

List buoys are used to make conceptual associations between the extended digits of the hand and various entities (Liddell 2003). The described entities and the extended digits of the hand blend, and those extended digits are then no longer only an index finger, a middle finger, etc., but visual representations of that entity. The ASL list buoys use the handshapes corresponding to those of the numeral signs from ONE through FIVE. As opposed to numerals, list buoys are usually produced with the weak hand, which is not held as upright as when producing a numeral sign, but tilted slightly to the side, and often held ahead of the chest rather than ahead of the shoulder. The type of list buoy constructions described by Liddell has been termed static list buoy by Vogt-Svendsen (personal communication 2003). 


\section{Static list buoys}

The discourse of the present study only contains one static list buoy, glossed in Example 1. It has three digits extended from the beginning, and is used to refer to three siblings (triplets), introduced as a group.

Example 1:

right hand SELF /TWIN touch-all-fingers ONE \#OF

left hand THREE/TWIN THREE-LIST--_---_---------

NON-1ST-PL-above-list touch-indexf OLDEST touch-indexf

BUT ONLY ONE KILO WEIGH BORN

BORN

She was one of a set of triplets, the oldest of them, but she only weighed one kilo when she was born...

The introduction of the siblings is done with a compound sign consisting of the one-handed form THREE (produced by the non-dominant hand, possibly anticipating the buoy) and the two-handed form TWIN (Figure 9a). The index finger is associated with the oldest sibling, and pointed at several times for reference to that sibling (Figure 9b), who is also the main character of the story. (In cases where there is an age difference between the referents, the index finger is used for the oldest referent). In the continued discourse, the list buoy on the weak hand is used as one of the active hands in two twohanded signs which are symmetrical in citation form; the signs BUT and KILO. The signs are produced with the citation form movement of both hands, but maintaining the handshape of the list buoy on the non-dominant hand, while the dominant hand uses the citation form handshape. Between those two signs, the list buoy handshape is maintained on the non-dominant hand while the dominant hand produces the two one-handed signs ONLY and ONE (Figure 9c). This only instance of a static list buoy co-occurs with $2 \%$ ( $n$ $=9$ ) of the one-handed signs. 

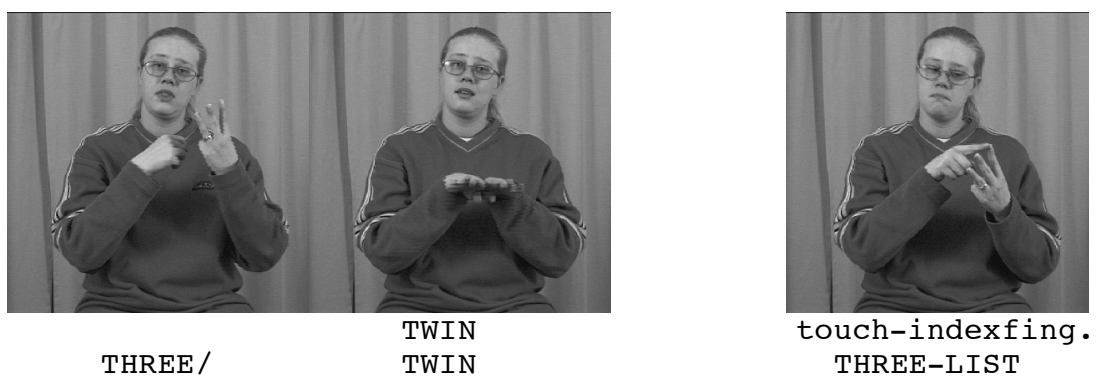

Figure 9a

Figure $9 b$

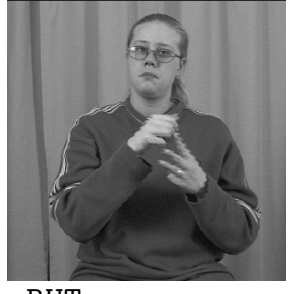

BUT

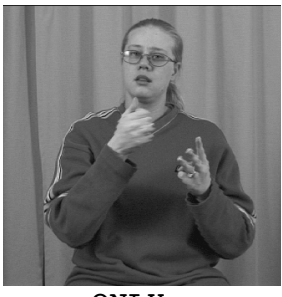

ONLY

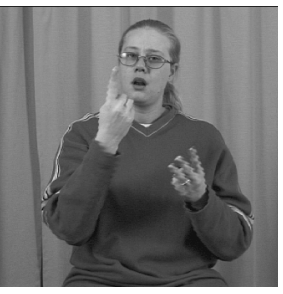

ONE

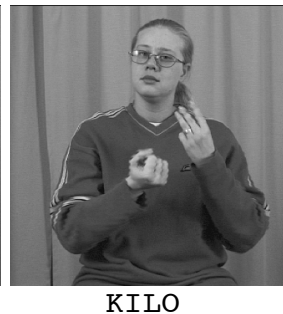

KILO

Figure 9c

Figure 9: Introduction of the referent 'triplets', returning to 'the oldest sibling' and use of the static THREE-LIST in the phrase 'but she only weighed one kilo'.

\section{Sequentially built list buoys}

The discourse that the present study focuses on mainly contains sequentially built list buoys, where a list is gradually built, with a maximum of five referents. Figure 10 shows a sequentially built list buoy. Note that the first six signs are not illustrated with photos, but are only glossed.

Sequentially built list buoys can serve the purpose of keeping track of referents that are described as they are enumerated. These entities are sometimes introduced in chronological order or some other logical order, but they may also simply be introduced in the order they come to the signer's mind. A particular referent can be kept constant by maintaining the handshape of the list buoy associated with it while the dominant hand produces onehanded signs. The referent can also be kept constant by maintaining the handshape of the list buoy on the non-dominant hand while it is used as base hand in an asymmetric two-handed sign or as one of the active hands in a symmetric sign (cf. Figure 10 below, the sign USA). The sequential list buoys in this discourse co-occur with $7 \%(n=44)$ of the instances of one-handed signs. 
NON-1ST-SING-f \#SHE-f PERF DO-RESEARCH WORLD DIFFERENT

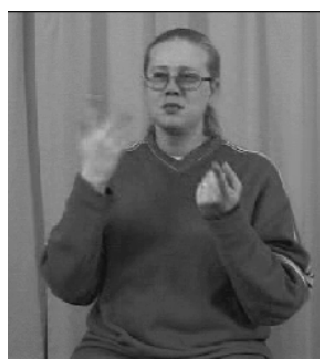

WHAT

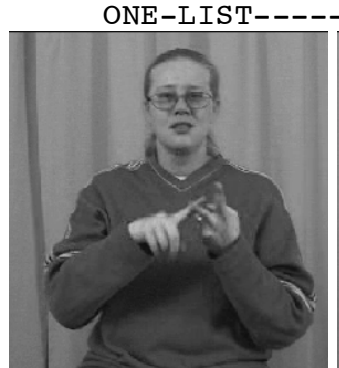

touch-middlefing

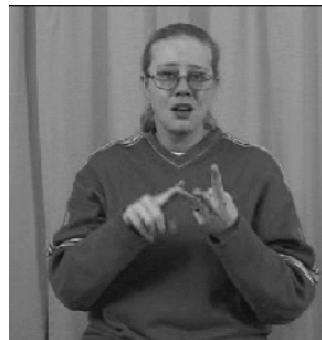

touch-ringfing

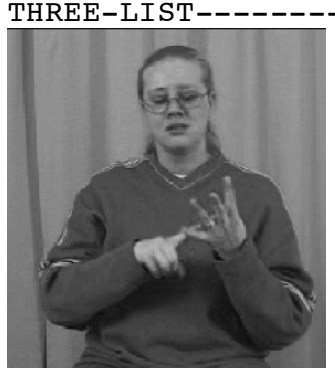

touch-littlefing

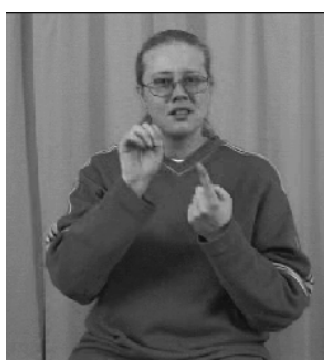

\#ASIA

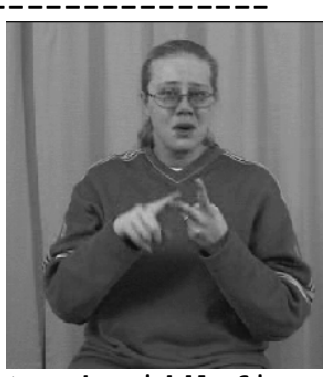

touch-middlefing

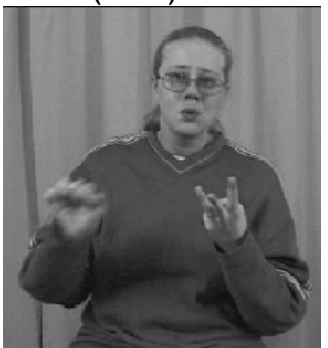

EUROPE

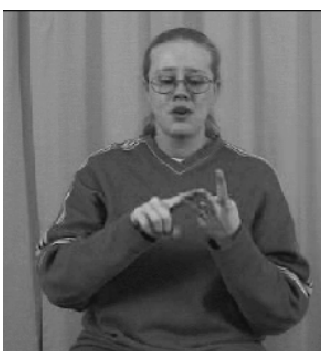

touch-ringfing

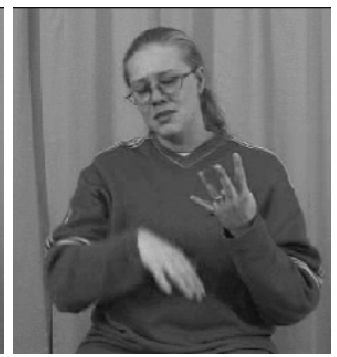

SOUTH

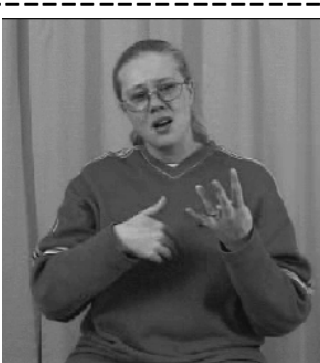

AUSTRALIA

She had done research in different countries all over the world - Asia, the US, Europe, down in Australia, and what have you.

Figure 10: Example of a sequentially built 'list buoy'. (NB! first line has no photos.) 
Sequentially built list buoys do not always have a specific referent associated with every digit, and having begun to produce what looks like a sequentially built list buoy, the signer may sometimes not continue to build it. In the discourse examined here, the signer associates, for example, ONE-LIST with the signs meaning 'her research' by almost touching it. Then she produces TWOLIST, which is followed by (and thus associated with) signs meaning 'her manner'. Finally, while she moves the right index finger to a fairly high starting position, she rapidly extends first the index finger, then the middle finger again, and lastly the other two fingers on the non-dominant hand (the preceding sign was a two-handed sign where both hands assumed the fist handshape) and then lets the dominant index finger move down - lightly touching the third and fourth finger of the non-dominant hand. This final sign sequence then, is used as a way of indicating that more referents could be given/named if needed, but that this is not deemed necessary for the specific purposes of the discourse.

\section{Point buoys}

Vogt-Svendsen and Bergman (this volume) describe another type of buoys referred to as point buoys, which they have identified in Norwegian Sign Language and Swedish Sign Language. There are two such buoys, POINT-G (index finger) and POINT-B (flat hand), which are described as a type of marker in space that help visualize temporal and spatial relationships between entities. The material of this study contains one example of the POINTG buoy, representing a point in time viz. 'the time that you are going to die'. It can be seen in Figure 5 above, where it is the base hand of GET-NEARER and SOON, and then remains in place during the one-handed signs DIE and \#THEN. This single instance of a point buoy in the discourse co-occurs with approximately $0.33 \%(n=2)$ of the one-handed signs.

\section{Occurrences of buoys in the material}

As has been noted in the descriptions of Swedish Sign Language buoys above, some buoys can occur with both one-handed and two-handed signs, whereas others will be replaced by a two-handed sign and may then reappear. When using list buoys, the digit associated with a particular referent can be touched both before and/or after that referent is mentioned, which means that their frequency can become very high. That is, because the signer may touch her finger both before and after articulating a referent, for every referent the list buoy may be touched twice. It is also typical that buoys are held for longer or shorter stretches of signing. We will therefore consider the number of sequences where buoys occur, in addition to the already presented number of one-handed signs they co-occur with. There are a total of six sequences where the POINTER buoy is used and another six sequences where 
the THEME buoy is clearly used. There are five sequences in the material where long lists are built, but there is only one static list buoy, and one point buoy. Together, these sequences with buoys co-occur with a total of $17 \%(n$ $=107$ ) of the one-handed signs. 


\section{Dominance reversals}

The range of buoys described in the previous section are all typically produced with the non-dominant hand, while the dominant hand continues to produce other signs. The discourse examined also contains instances of onehanded signs produced with the non-dominant hand, where use of the dominant hand might have been expected and where no sign co-occurs on the dominant hand. For want of a better term, this is referred to as 'dominance reversal'.

In the whole discourse, only $1 \%(n=8)$ of the one-handed signs are produced by the non-dominant hand. ${ }^{10}$ Seven of them are pointing signs (INDEX$\mathrm{x})$ produced with the non-dominant hand, and no sign co-occurring on the dominant hand. Five of those pointing signs occur at the end of a signed sequence and are followed by both hands assuming a two-handed variant of 'at chest', where the fist of the non-dominant hand is clasped by the dominant hand in front of the signer's chest, and the forearms rest against the body. Two of the instances of INDEX-x produced with 'dominance reversal' and followed by both hands in the 'at chest' position occur in the transcribed Examples 2 and 3.

Example 2:

PLUS NON-1ST-SING-1 GLADLY MUCH DISCUSS WITH PERSON

PLUS GLADLY DISCUSS WITH

PATIENT OBJ.pl DIE PATIENT

PATIENT PATIENT

WILL ON WAY DIE at chest

WILL ON WAY DIE INDEX-f-fl at chest

And she enjoys discussing a lot with patients who are going to die.

\footnotetext{
${ }^{9}$ Frishberg (1985:79) uses the term in her study of hand preferences in signed languages and "the coordinated behaviour of the two hands within the flow of signs in running discourse". According to Frishberg, dominance reversal is never obligatory, but used to create semantic connections or contrasts within a narrative.

${ }_{10}$ Producing a sign with the non-dominant hand as the active hand does not change the signer's hand dominance, and dominance reversal is thus in a sense actually a misnomer.
} 
Example 3:

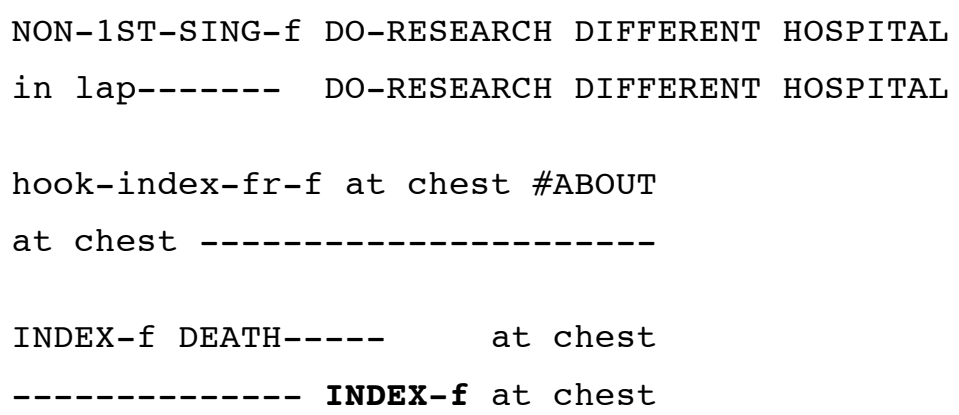

She has done research in many different hospitals - about death.

The other two of the seven instances of pointing signs produced with 'dominance reversal' are not followed by both hands being placed in the 'at chest' position. They are both instances of NON-FIRST-PL and co-occur with the signer changing perspective, which could be regarded as the end of something and/or a kind of contrast.

The use of 'dominance reversal' seems to be part of how the signer organizes the discourse structure, marking the end of something. It has earlier been noted for Swedish Sign Language that the end of a 'paragraph' can be marked by a one-handed sign where the signer switches active hand (Brita Bergman, personal communication).

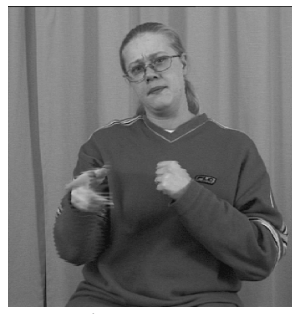

NON-1ST-SING

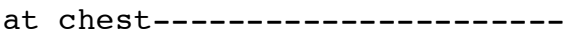

That was what she wanted. The nurse said "No, wait..."

Figure 11: 'Dominance reversal' in constructed dialogue.

There is one sequence in the material where change of active hand in a onehanded sign is used to indicate change of perspective and constructed dialogue (cf. Figure 11 above). Here, the signer describes something a dying patient wants to do, and produces NON-1ST-SING-f WANT-TO from the signer's perspective. Then a comment directed to that patient from a nurse is recounted. The signer switches from signer's perspective to the nurse's perspective using 'dominance reversal', but also by changing the position of her 
head (as seen in Figure 11). First, she produces the one-handed sign NO with the non-dominant hand. That one-handed sign then remains on the nondominant hand (cf. the section above about sign fragments keeping the referent constant) while the rest of the nurse's comment, the one-handed sign WAIT, is produced with the dominant hand. ${ }^{11}$ Interestingly, this 'reversal of roles' for the hands not only means that the non-dominant hand produces one-handed signs. The dominant hand takes on the role of the non-dominant hand, and is either held in the 'at chest' position, produces instances of assimilation, or (as we saw in Figure 11) it produces a 'sign fragment'.

\footnotetext{
${ }^{11}$ The perseveration of WANT-TO on the dominant hand, in the third photo, may be due to the fact that the next sign to be produced by that hand uses the same handshape and place of articulation.
} 


\section{Discussion}

In the introduction, it was mentioned that there are several factors contributing to a markedly two-handed impression of this stretch of signed discourse. As we have seen, looking only at whether a sign is one-handed or twohanded in citation form yields a distribution that is roughly fifty-fifty.

However, if we also include the activities of the non-dominant hand when not part of a two-handed sign, the impression we get is quite different. In Figure 12, a summary of what the non-dominant hand does when it is not part of a two-handed sign is illustrated as a continuum. ${ }^{12}$ At the bottom left of the continuum are those instances where the non-dominant hand is least active, and where it does not contribute to the content of the discourse. The farther to the right and up, the more active the non-dominant hand is, and the more it contributes to the discourse production and to the content of the discourse.

dominance reversals (1\%)

buoys $(17 \%)$

sign fragments (6\%)

doubling $(2 \%)$

mirroring $(4 \%)$

mirror at chest $(6 \%)$

at chest $(22 \%)$

in lap (19\%)

Figure 12: Continuum of the activities of the non-dominant hand.

In the $19 \%(n=116)$ of instances of one-handed signs that co-occur with 'in lap', the non-dominant hand is maximally inactive, and does not take part in the discourse production at all. But, in the two rest positions - 'at chest' $(22 \%, n=133)$ and 'mirror at chest' $(6 \%, n=37)$ - the non-dominant hand

\footnotetext{
${ }^{12}$ Assimilation, which is part of the production of a two-handed sign, has not been included in the continuum.
} 
is held at chest level, where many one-handed and two-handed signs are produced. Due to this, the non-dominant hand appears more involved in the discourse production than it does when it rests in the signer's lap, which is also true for one-handed signs co-occurring with 'mirroring' $(4 \%, n=22)$. Adding up these activities of the non-dominant hand, we find that $32 \%$ of the occurrences of one-handed signs in the discourse $(n=192)$, are produced with the non-dominant hand held in 'at chest', 'mirror at chest' or producing an instance of 'mirroring'. In these, the non-dominant hand does not contribute to the content of the discourse, and in a majority of the cases it does not move, but contributes to the two-handed impression of the sign production.

There are also a large number of instances of assimilation in the discourse, where the non-dominant hand is either anticipating or perseverating from a preceding or following two-handed sign, while the dominant hand is producing a one-handed sign. Assimilation co-occurs with $23 \%(n=141)$ of the one-handed signs in the discourse. Even though assimilation is a result of the production of two-handed signs, it partly occurs during the production of one-handed signs and thus contributes to the impression of two hands being used simultaneously.

The use of the non-dominant hand in the present Swedish Sign Language discourse seems very different from what Klima \& Bellugi (1979:217) claim regarding signed ASL discourse. According to them, "[i]n signed discourse, the base hand as a location frequently lags behind the active hand", and it is only in lexicalized compounds that anticipation is typical, rather than this delay. They also quote an unpublished manuscript by Friedman (1974), stating that the "hand configuration of the articulator of a given sign is formed first, and only then is the handshape of the place of articulation hand shaped or even brought into the signing space". ${ }^{13}$ The non-dominant hand as place of articulation is ready at the same time as the dominant hand in this discourse, or anticipates it, also in signs that are not lexicalized compounds.

Turning to instances where content is added and the non-dominant hand is active in the signing, a total of $25 \%(n=159)$ of the one-handed signs cooccur with 'doubling', 'sign fragments' or different buoys. 'Dominance reversal' per definition means that the non-dominant hand is active. In the $1 \%$ $(n=8)$ of one-handed signs in the discourse that occur with 'dominance reversal', the dominant hand is either held in 'at chest' position, or assimilates with at preceding or following sign, or produces a 'sign fragment'. This reversal of roles also gives a two-handed impression.

Taken all together, a total of approximately $90 \%(n=1038)$ of the 1151 instances of signs in the discourse are thus produced in a way that gives an impression of two hands participating simultaneously.

\footnotetext{
${ }^{13}$ The quote from Klima \& Bellugi (1979:391) is in Note 12, Chapter 9, and refers to Friedman (1974). The list of references, however, lists Friedman 1974a and 1974b. They are both for unpublished manuscripts and I have not been able to tell which one is intended.
} 
There is another interesting tendency in the material, however, which for parts of the discourse could be regarded as working against the impression of two-handedness discussed above. As mentioned earlier there are seven longer sequences where the non-dominant hand frequently rests in the 'in lap' position. During these sequences, the non-dominant hand is only used for a few instances of list buoys, assimilation and 'mirroring'. In general, though, the sequences give a markedly one-handed impression of the signing. 'In lap' co-occurs with $19 \%(n=116)$ of the one-handed signs. (These, in turn, constitute the $10 \%$ of the signs in the discourse that do not give a two-handed impression.)

When the non-dominant hand adds content to the discourse, without being part of a two-handed sign, it often seems to help indicate to the addressee who/what the speaker is currently talking about. This is done in a number of different ways. The non-dominant hand can remain in place from a previous symmetrical two-handed sign as a 'sign fragment', thus maintaining the topic of the discourse. The different buoys in the discourse - the POINTER buoy, the THEME buoy, list buoys and point buoys - also help the listener understand who or what the current referent(s) is/are and the relationships between them, or that the referent is an important discourse theme. Finally, 'dominance reversal' seems to be part of how the signer structures the discourse, marking e.g. the beginning of something new or change of perspective.

The present study provides us with more knowledge about simultaneity and the use of the non-dominant hand in Swedish Sign Language through the analysis of one signed piece of discourse. To find out more, we will have to analyze the use of the non-dominant hand in other kinds of discourse; monologues as well as dialogues, different styles and registers, and produced by different signers. As for the rest position, 'in lap', its existence is a consequence of the signer sitting down, and we do not know what happens if the signer is standing up. Analyzing more texts will also help us to better understand the use and meaning of 'mirroring', 'doubling', 'sign fragments', buoys, and reverse dominance. The existence, and to some extent also the frequency, of the rest positions, as well as their contribution to the twohanded impression that is so apparent in this signed discourse is of interest to others as well as to linguists. For example, for teachers of Swedish Sign Language, this can be of practical use to, helping them give their students more accurate advice than: "You need to use the other hand more". 


\section{Summary}

In this chapter, the activities of the non-dominant hand when not participating in the production of a two-handed sign have been described for one complete Swedish Sign Language discourse. These activities can be seen as a continuum (cf. Figure 12 above), starting from the bottom left with the activities where it is least active. First, there is a position where the nondominant hand is resting 'in lap'. Then there are rest positions were the nondominant hand looks more active, either held in the position 'at chest' where it is not influenced by the dominant hand, or in 'mirror at chest' where it mirrors the handshape and orientation of the non-dominant hand. In these positions, the non-dominant hand is still not actually moving, and does not add to the content of the discourse.

Next, the non-dominant hand begins to move and there are instances where the non-dominant hand mirrors the actions of the dominant hand when it produces one-handed signs. In 'mirroring' the handshape, orientation, and movement of the dominant hand are mirrored, but at a slightly lower location. This is a sign-internal, purely phonetic process, which does not add content to the discourse. In 'doubling', both hands are active, performing the same movement at the same height. Here, the non-dominant hand also begins to add content to the discourse, reinforcing what is said. Producing a 'sign fragment', the non-dominant hand remains in place after having been part of the production of a two-handed sign, and is held while the dominant hand produces signs, telling us who/what the topic of the discourse is.

In the top right corner of the continuum, the non-dominant hand independently produces signs. There are various kinds of 'buoys', which are normally produced by the non-dominant hand while the dominant hand also produces signs. All of the buoys contribute their own meaning to the discourse. Finally, in 'dominance reversals', a one-handed sign is produced with the non-dominant hand without any sign being produced simultaneously by the dominant hand. Dominance reversals seem to be part of how the signer organizes the discourse, marking, for example, the end of something.

Thus $90 \%$ of the signs in this discourse give the impression that both hands are being used simultaneously, when all the activities of the nondominant hand are taken into consideration. 


\section{References}

Ahlgren, Inger \& Brita Bergman. 1994. "Reference in Narratives". In Inger Ahlgren, Brita Bergman, \& Mary Brennan (Eds.), Perspectives on Sign Language Structure: Papers from the Fifth International Symposium on Sign Language Research Vol. 1, 29-36. Durham: International Sign Linguistics Association.

Bergman, Brita. 1983. "Verbs and Adjectives: Some Morphological Processes in Swedish Sign Language". In Jim G. Kyle \& Bencie Woll (Eds.), Language in Sign: An International Perspective on Sign Language, 3-9. London/Canberra: Croom Helm.

Battison, Robbin. 1974. "Phonological Deletion in American Sign Language". Sign Language Studies, 5, 1-19.

Emmorey, Karen (Ed.). 2003. Perspectives on Classifier Constructions in Sign Languages. Mahwah, $\mathrm{NJ} /$ London: Lawrence Erlbaum.

Friedman, Lynn A. 1975. "Space, Time and Person Reference in American Sign Language". Language, 51(4), 940-961.

Frishberg, Nancy. 1985. "Dominance Relations and Discourse Structures". In William C. Stokoe \& Virginia Volterra (Eds.), SLR'83 Proceedings of the III. International Symposium on Sign Language Research. Rome, June 22-26 1983, 79-90. Silver Spring, MD: Linstok Press Inc. and Rome, Italy: Instituto di Psicologia CNR.

Gee, James Paul \& Judy Ann Kegl. 1983. "Narrative/Story Structure, Pausing, and American Sign Language". Discourse Processes, 6, 243-258.

Klima, Edward S. \& Ursula Bellugi. 1979. The Signs of Language, with Robbin Battison, Penny Boyes Braem, Susan Fischer, Nancy Frishberg, Harlan Lane, Ella M. Lentz, Don Newkirk, Elissa L. Newport, C. C. Pedersen, \& Patricia Siple. Cambridge, MA: Harvard University Press.

Liddell, Scott K. 2003. Grammar, Gesture, and Meaning in American Sign Language. Cambridge: Cambridge University Press.

_- Marit Vogt-Svendsen, \& Brita Bergman. 2004. Crosslinguistic Comparison of Buoys: Evidence from American, Norwegian and Swedish Sign Language. Paper presented at 26 Jahrestagung der Deutschen Gesellschaft für Sprachwissenschaft, an der Johannes- Gutenberg-Universität Mainz vom 25-27 Februar 2004.

Miller, Christopher. 1994. "Simultaneous Constructions in Quebec Sign Language". In Mary Brennan \& Graham H. Turner (Eds.), Word-order Issues in Sign Language. Working Papers, 89-112. Durham: International Sign Linguistics Association.

Sandler,Wendy. 1993. "Hand in Hand: The Roles of theNon-dominantHand in Sign Language Phonology". The Linguistic Review, 10(4), 337-390.

Svenskt teckenspråkslexikon. 1997. Leksand: Sveriges Dövas Riksförbund.

Wallin, Lars. 2000. "Two Kinds of Productive Signs in Swedish Sign Language: Polysynthetic Signs and Size and Shape Specifying Signs”. Sign Language \& Linguistics, 3(2), 237-256. 